\title{
La Fuerza Multilateral y la multiplicidad nuclear en la OTAN (1960-1965)
}

\section{Multilateral Force and NATO’s nuclear multiplicity (1960-1965)}

\author{
JOSÉ RAMÓN CUMPLIDO MUÑOZ ${ }^{1}$ \\ Universitat de València \\ jrcumplido@yahoo.es
}

\begin{abstract}
Resumen: Desde finales de la década de 1950 y hasta 1965, el gobierno de Estados Unidos (EEUU) promovió la creación de una fuerza naval armada con misiles balísticos, conocida como Fuerza Multilateral (Multilateral Force, MLF), con tripulaciones multinacionales y bajo mando de la Organización del Tratado del Atlántico Norte (OTAN). La propuesta inicial de la administración Eisenhower trataba de satisfacer las quejas de algunos miembros de la OTAN acerca del monopolio que EEUU ejercía sobre la defensa nuclear de Europa. Aunque oficialmente se buscaba proporcionar un papel activo a los países europeos, en particular Alemania, en la estrategia nuclear conjunta, con la MLF los EEUU confiaban en inducir a que tanto el Reino Unido como Francia integraran sus respectivos componentes disuasorios en un único organismo que, aunque no estuviera bajo el control directo estadounidense, al menos evitara estrategias nucleares independientes, cuando no divergentes.
\end{abstract}

Palabras clave: Fuerza Multilateral, disuasión nuclear, Guerra Fría, OTAN, misil Polaris.

Abstract: In the late 1950s and until 1965, the US government promoted the creation of a naval force armed with ballistic missiles, known as Multilateral Force (MLF), with multinational crews and under NATO command. The initial proposal of the Eisenhower administration was to satisfy complaints from some NATO members about the US monopoly on European nuclear defense. Although the official aim was to provide an active role to European countries, particularly to Germany, in the joint nuclear strategy. with the MLF, the US were relying on both UK and France to integrate their respective nuclear deterrents into a single organism. However, this one would not be under the direct US control, at least it will avoid independents, if not divergents, nuclear strategies.

Keywords: Multilateral Force, nuclear deterrence, Cold War, NATO, Polaris missile.

${ }^{1}$ Las traducciones del inglés y del francés son nuestras.

Recibido: 31 de octubre de 2016; aceptado: 20 de marzo de 2017; publicado: 21 de septiembre de 2017.

Revista Historia Autónoma, 11 (2017), pp. 241-259.

e-ISSN: 2254-8726; DOI: https://doi.org/10.15366/rha2017.11.012 
Introducción

Al inicio del período de la Guerra Fría, pocos años después de concluir la Segunda Guerra Mundial, los devastados países europeos carecían de fuerzas militares con las que hacer frente a una temida invasión soviética. El único factor que podía contrarrestar la superioridad numérica de la Unión de Repúblicas Socialistas Soviéticas (URSS), era el arsenal nuclear estadounidense; gracias a este factor, los EEUU trazaron en Europa la estrategia más conveniente a sus necesidades. Esta situación, muy cercana a un protectorado militar, fue aceptada inicialmente por los gobiernos europeos, cuyos esfuerzos se encaminaban a la reconstrucción.

Esta complacencia quedó rota en 1956, cuando Francia y Gran Bretaña se vieron forzadas a retirar sus tropas de Suez ante las amenazas de la URSS de utilizar armas atómicas, lo que demostraba que EEUU no necesariamente usaría su arsenal nuclear para respaldar los intereses europeos ${ }^{2}$. Para paliar el desaire, el presidente Eisenhower anunció en julio de 1957 su intención de estacionar armas nucleares en Europa con las que contrarrestar los misiles de alcance intermedio (Intermedium Range Balistic Missile, IRBM) soviéticos y, en claro gesto compensatorio hacia su más firme aliado, anunció la venta de IRBM al Reino Unido ${ }^{3}$. Sin embargo, el uso de estas armas requería la autorización estadounidense, por lo que algunos aliados expresaron la amargura de sentirse rehenes de una situación en la que en una guerra nuclear entre superpotencias, pero sobre suelo europeo, no tendrían capacidad de decisión ${ }^{4}$.

Este temor quedaría invertido el 4 de octubre, con la puesta en órbita del satélite Sputnik I, cuyo lanzador, el cohete 8K71PS, no se molestaba en ocultar sus posibilidades militares, convirtiéndose en 1959, efectivamente, en el primer misil balístico intercontinental (Intercontinental Ballistic Missile, ICBM) en servicio. Con el territorio estadounidense al alcance de estos misiles, para los que por entonces no se disponía de un equivalente, el temor de los aliados pasó a ser que EEUU vacilara en el uso de su arsenal nuclear para defender Europa.

Para despejar los temores sobre el resurgimiento del aislacionismo estadounidense, Eisenhower reiteró en diciembre, durante la reunión de los líderes de la OTAN en París, el firme compromiso en la defensa de Europa; previamente, a comienzos de noviembre, el secretario de Estado, John Foster Dulles, ofreció ante el Consejo de Atlántico Norte (North Atlantic Council, NAC) el despliegue de IRBM en Europa. Estos misiles estarían sujetos al sistema de lanzamiento mediante dos llaves ${ }^{5}$; mientras los europeos detentarían la propiedad de los misiles instalados en su territorio, EEUU custodiaría las cabezas nucleares. Si se acordaba su utilización, estas

\footnotetext{
${ }^{2}$ Kustnetsov, Evgeny, "The Multilateral Force debates". «http://www.cvce.eu/obj/evgeny_kustnetsov the multilateral force debatesen-937a5818-7fea-47da-44e-11114da4e0a3.html» [consultado el 6 de febrero de 2017]. ${ }^{3}$ Withers, Christopher, The multilateral force: an analysis and presentation of United States policy and of the reactions of the principal European powers, tesis doctoral, The American University, 1966, p. 5

${ }^{4}$ Ibídem, p. 5.

${ }^{5}$ Nash, Philip, The Other Missiles of October: Eisenhower, Kennedy, and the Jupiters, 1957-1963, Chapel Hill, University of North Carolina Press, 1997, pp. 18-19.
} 
se entregarían al país anfitrión, que se encargaría de armar el misil; finalmente, dos oficiales, uno estadounidense y otro del país aliado, poseedores cada uno de una llave de seguridad, activarían el misil para su lanzamiento. Sin embargo, únicamente Gran Bretaña aceptó en 1958 el ofrecimiento, seguida por Turquía e Italia en 1959. Francia, por su parte, rechazó cualquier acuerdo que implicara la renuncia al control sobre misiles o cabezas nucleares presentes en su territorio.

Este rechazo fue interpretado como el deseo europeo de implicarse más intensamente en la defensa nuclear de la OTAN; ante la eventualidad de que EEUU no compartiera con sus aliados un mayor grado de control sobre las armas nucleares, lo más probable es que aquellos derivaran hacia la neutralidad o que desarrollaran una capacidad nuclear propia ${ }^{6}$. Ambos supuestos implicaban un debilitamiento de la seguridad de EEUU y de la cohesión de la OTAN; como alternativa, surgió entonces la idea de constituir una fuerza nuclear conjunta en la que pudieran participar los aliados europeos, antes de que estos encontraran una solución que quedase fuera del control estadounidense? ${ }^{7}$.

En agosto de 1959, el general Norstad, Comandante Supremo Aliado en Europa (Supreme Allied Commander in Europe, SACEUR) refinaba la idea mediante la creación de una fuerza de misiles de alcance medio (Medium Range Balistic Missile, MRBM), a disposición de la OTAN y bajo su control, de forma que la alianza pudiera considerarse una "cuarta potencia nuclear".

\section{La pérdida del monopolio nuclear estadounidense}

Para EEUU era esencial mantener el control sobre todas las armas nucleares implicadas en un hipotético conflicto con la URSS, lo que implicaba evitar la propagación de fuerzas nucleares independientes. Sin embargo, y en contradicción con esta directriz, EEUU proveyó asistencia técnica decisiva en el programa nuclear británico. Efectivamente, el 3 de octubre de 1952 el Reino Unido detonó un dispositivo de fisión en las islas Montebello, en Australia. Salvo el material fisible, de producción autóctona, el dispositivo era una copia de Fat Man (el arma empleada sobre Nagasaki), circunstancia que presagiaba la posterior dependencia.

Las primeras armas nucleares británicas consistieron en bombas de caída libre operativas en 1954 con los bombarderos de la V-Force. Sin embargo, ante la potenciación de las defensas antiaéreas soviéticas, se estimaba que estos bombarderos resultarían inútiles hacia 1965. EEUU propuso como alternativa un programa conjunto por el que asumirían el desarrollo de un ICBM, mientras que el Reino Unido haría lo propio con un MRBM. Aunque el diseño de este, de

\footnotetext{
${ }^{6}$ Withers, Christopher, The multilateral force... op. cit., p. 8.

${ }^{7}$ Schlesinger, Arthur Meier, A Thousand Days: John F. Kennedy in the White House, Boston, Houghton Mifflin Harcourt, 2002, p. 850.

${ }^{8}$ Withers, Christopher, The multilateral force... op . cit., p. 8.
} 
nombre Blue Streak, concluyó en 1957, el proyecto fue cancelado debido a la certeza de que cualquier silo emplazado en las islas británicas no dispondría de tiempo de reacción ante un ataque soviético.

A cambio de la construcción de una base de submarinos en Escocia, EEUU ofreció la incorporación del Reino Unido a uno de sus programas de MRBM: el Polaris, lanzado desde submarinos, y el Skybolt, lanzado desde bombarderos; Harold MacMillan acordó su participación en este último y el establecimiento de la base estadounidense ${ }^{9}$. Mediante este acuerdo, firmado en junio de 1960, el Reino Unido estableció el Skybolt como su principal vector nuclear ${ }^{10}$, comprometiéndose a adquirir 144 misiles y financiar las modificaciones para ser lanzados por los bombarderos de la $V$-Force ${ }^{11}$.

En Francia, de forma paralela, los informes del Commissariat à l'énergie Atomique llevaron al general Pierre Gallois a concluir que era posible para Francia dotarse de armas nucleares. En 1958 el presidente del gobierno francés, Félix Gaillard, aprobó la creación de una fuerza nuclear, la Force de Frappe, según las consideraciones estratégicas de Gallois ${ }^{12}$. Este componente nuclear representaba para el presidente de la república, Charles De Gaulle, la recuperación de la soberanía nacional perdida por los europeos al delegar su defensa en la OTAN; De Gaulle consideraba, además, que Washington utilizaba la alianza para controlar "la defensa y la política exterior, e incluso, el territorio de sus aliados"13. Finalmente, el 13 de febrero de 1960 Francia hizo estallar su primera bomba atómica, Gerboise bleue, en el campo de pruebas de Reggane (Argelia).

Aparecía así en escena un actor que no estaba dispuesto a seguir la batuta estadounidense; aún más, se temía que este ejemplo supusiera un aliciente para que Alemania buscara su propia fuerza nuclear. Aunque Adenauer había renunciado en 1954 al desarrollo de armas nucleares, los conservadores alemanes, a riesgo de despertar "recelos del pasado", contemplaban las restricciones en materia nuclear como una "indiscriminada prolongación de Versalles y Yalta"14, que no estaba en consonancia con la renovada presencia alemana en Europa.

En marzo el general Norstad propuso la creación de una fuerza nuclear compuesta por EEUU, Gran Bretaña y Francia, bajo el mando de un comandante no necesariamente estadounidense y a disposición de la OTAN. Randolph Burgess, representante de EEUU en el NAC, aceptó oficialmente la propuesta, aunque enfatizó como condición que EEUU mantuviera el control de las cabezas nucleares, lo que, en última instancia, era inaceptable para Francia.

\footnotetext{
${ }^{9}$ MacMillan, Harold, Memoirs. Vol. 5, Londres, MacMillan, 1972, pp. 254-255.

${ }^{10}$ Grove, Eric, Vanguard to Trident: British Naval Policy Since World War II, Annapolis, Naval Institute Press, 1987, p. 234.

${ }^{11}$ Brookes, Andrew, V-Force. The History of Britain's Airborne Deterrent, Londres, Jane's, 1982, p. 114.

${ }^{12}$ Vid. Gallois, Pierre, Stratégie de l'âge nucléaire, París, Calmann-Lévy, 1960.

${ }^{13}$ De Gaulle, Charles, Discours et messages. Vol. 4, París, Plon, 1970, p. 199.

${ }^{14}$ Schrafstetter, Susanna, "The Long Shadow of the Past: History, Memory and the Debate over West Germany's Nuclear Status, 1954-69", en History \& Memory, vol. 16, 1 (2004), p. 138.
} 
En la búsqueda de una fuerza nuclear común, el secretario de Defensa, Thomas S. Gates, propuso en abril la venta o la fabricación conjunta de Polaris con el compromiso de no ser destinados a una fuerza independiente; la propuesta incluía modificar el sistema de doble llave y entregar el control de la cabeza nuclear y el armado del misil al SACEUR ${ }^{15}$. La contrapropuesta de De Gaulle, por su parte, incluía el control sobre la totalidad de las cabezas nucleares francesas y sobre al menos un tercio de los misiles ${ }^{16}$. Aunque los EEUU deseaban la instalación de parte de los misiles en Francia, no iban a ceder su control, por lo que se hacía perentoria una propuesta que soslayara estos problemas. El Departamento de Estado propuso la creación de una flota de submarinos armados con Polaris, cuya propiedad sería detentada por la OTAN. El general Norstad, cuya anterior propuesta contemplaba emplazamientos terrestres, admitió que "desde el punto de vista militar [...] un despliegue naval de los misiles ofrecía ciertas ventajas" ${ }^{17}$.

\section{El "informe Bowie"}

El 1 de agosto de 1960 Robert Bowie, que había trabajado en el Departamento de Estado entre 1953 y 1957 y por entonces director del Centro de Estudios Internacionales de Harvard, entregó al Departamento de Estado un informe titulado The North Atlantic Nations: Tasks for the 1960s (desclasificado en $1989^{18}$ ) en el que analizaba los retos que la OTAN debía afrontar durante la siguiente década en materia nuclear.

El escenario en el que EEUU podía ser atacado únicamente mediante bombarderos soviéticos había sido sustituido por otro en el que el territorio estadounidense era vulnerable a los ICBM, lo que dejaba en entredicho su credibilidad para usar armas nucleares en defensa de Europa. Por otra parte, la recuperación económica de Europa y su búsqueda de una unidad política, animaba las esperanzas de los europeos de lograr un mayor reconocimiento internacional y desempeñar un papel proporcional en la estrategia común. Bowie concluía que se debía otorgar a Europa cierto control sobre su defensa, aunque evitando la proliferación de programas nucleares nacionales. Para satisfacer estos términos contradictorios, Bowie proponía mejorar las capacidades convencionales de la OTAN para contrarrestar una agresión soviética sin recurrir a las armas nucleares ${ }^{19}$. Al mismo tiempo, EEUU debería dotar a la OTAN de un componente nuclear conjunto, pues el caso del Reino Unido demostraba que ningún país

\footnotetext{
${ }^{15}$ Trachtenber, Marc, A constructed peace: the making of the European settlement, 1945-1963, Princeton, Princeton University Press, 1999, p. 212.

${ }^{16}$ Withers, Christopher, The multilateral force ... op. cit., p. 9.

${ }^{17}$ Watson, Robert, History of the Office of the Secretary of Defense. Vol. 4: Into the Missile Age 1956-1960, Washington, Office of the Secretary of Defense, 1988, p. 552

${ }^{18}$ Bowie, Robert Richardson, The North Atlantic nations tasks for the 1960's: a report to the Secretary of State, August 1960, College Park, University of Maryland, 1991.

${ }^{19}$ Ibidem, p. 38.
} 
europeo poseía recursos suficientes para desarrollar una disuasión independiente que, por otra parte, resultaría cara, redundante e introduciría factores de división en la OTAN ${ }^{20}$.

Bowie proponía crear una fuerza multinacional, formada en una primera etapa interina por cinco submarinos estadounidenses armados con Polaris ${ }^{21}$. En una segunda fase estos submarinos serían vendidos y pasarían a ser manejados por tripulaciones multinacionales ${ }^{22}$. Su financiación y propiedad sería común, de forma que ningún aliado pudiera retirar buques para emplearlos como fuerza propia. El uso de esta flota quedaría determinado por una autoridad superior al SACEUR en caso de un ataque nuclear a gran escala y por decisión del NAC en otras contingencias.

Bowie presentó su informe el 12 de septiembre; aunque se preveía que el misil Polaris no sería operativo antes de dos años, y la US Navy no dispondría de suficientes submarinos hasta pasado un período superior, la propuesta pareció satisfacer a Eisenhower y al secretario de Estado Christian Herter ${ }^{23}$. La Junta de Jefes de Estado Mayor de EEUU, por su parte, argumentó que la internacionalidad de las tripulaciones no era práctica y que la propiedad compartida de la flota dificultaría su gestión y complicaría la decisión de ordenar los lanzamientos. En su lugar, propuso una fuerza formada por los componentes nucleares europeos bajo el mando y control de la OTAN. Eisenhower rechazó estas objeciones y dio su aprobación a la propuesta de Bowie ${ }^{24}$.

El secretario Gates argumentaba en noviembre que el traspaso de armas nucleares a un organismo internacional requeriría de la aprobación del Congreso, extremadamente celoso del monopolio nuclear estadounidense, aunque "pareciera ignorar que ese monopolio se había perdido" 25 . Herter presentó la propuesta el 16 de diciembre en la reunión ministerial de la OTAN en París. En su discurso, se comprometió a aportar cinco submarinos armados con Polaris para formar una fuerza nuclear multilateral, condicionada a que los aliados adquirieran aproximadamente 100 misiles.

El gobierno británico dudaba de la necesidad y utilidad de una fuerza semejante. Por otra parte, se consideraba que la propiedad compartida de los buques encarecería el coste, mientras se temía que se pudieran transferir conocimientos, tecnología o armas a potencias que por entonces no eran nucleares. Ahondado en seculares desencuentros, se dudaba de la voluntad francesa de renunciar a su programa nuclear al que consideraba "origen de la propuesta estadounidense" 26 . Sin embargo, el Foreign Office señalaba la conveniencia de participar en esta fuerza, dado que no se podía permitir una disminución de la influencia británica en la

\footnotetext{
${ }^{20}$ Ibídem, p. 38.

${ }^{21}$ Ibídem, p. 60.

${ }^{22}$ Ibídem, pp. 61-63.

${ }^{23}$ Watson, Robert: History of the Office... op. cit., pp. 553-554.

${ }^{24}$ Noble, George Bernard, Christian A. Herter, Nueva York, Cooper Square Publishers, 1970, p. 260.

${ }^{25}$ Ferrell, Robert Hugh (ed.), The Eisenhower diaries, Nueva York, Norton, 1981, p. 381.

${ }^{26}$ Pagedas, Constantine, Anglo-American strategic relations and the French problem, 1960-1963: a troubled partnership, Londres, Frank Cass, 2000, p. 117.
} 
OTAN (asunto acuciante con la aparición de Francia como potencia nuclear) y a cualquier escollo con EEUU. Aunque la fuerza nuclear británica había proporcionado al país una renovada influencia internacional, comenzaba a revelar sus carencias. En primer lugar, era dependiente de la tecnología norteamericana, en especial de los vectores de lanzamiento, mientras que el programa de ICBM estadounidense situaba a la $V$-Force en un aspecto marginal en la defensa occidental. Por último, se reconocía que la defensa frente a la URSS quedaba cubierta mediante la disuasión nuclear norteamericana, por lo que no existía una necesidad específica de una contribución británica. El Reino Unido debía comprometerse en la creación de un sistema en el que pudiera emplazar la totalidad de su fuerza nuclear como gesto de solidaridad hacia Europa, animando a Francia a actuar de idéntico modo y convenciendo a Alemania y otros países europeos de lo innecesario de sus esfuerzos por convertirse en potencias nucleares ${ }^{27}$. Por otra parte, el PIB británico había disminuido, por lo que resultaría imposible contribuir a esta fuerza y mantener los compromisos con la Commonwealth y la OTAN. Macmillan acabó aceptando la fuerza multilateral como mal menor, priorizando el componente disuasorio conjunto occidental y a que EEUU continuara comprometido en la defensa de Europa.

\section{Kennedy como promotor de la MLF}

Tras asumir la presidencia, Kennedy no trató las cuestiones nucleares hasta la visita del canciller Adenauer a Washington el 12 y 13 de abril de 1961, cuando reafirmó su compromiso con la defensa de Europa y que se encontraba convencido del pleno apoyo de Alemania al concepto de la fuerza multilateral. En un Memorando de Acción de Seguridad Nacional (National Security Action Memorandum, NSAM) del 24 de abril ${ }^{28}$, Kennedy aclaraba su postura respecto a la creación de una fuerza multilateral e indicaba su intención de destinar a la OTAN de forma permanente cinco submarinos.

La primera mención oficial a la fuerza multilateral (Multi-Lateral Force, MLF) se produjo durante el discurso de Kennedy ante el Parlamento canadiense ${ }^{29}$. En primer lugar, Kennedy anunció que priorizaría las capacidades convencionales y que animaría a los aliados en el mismo sentido. En lo que se refiere a la disuasión nuclear, constituiría un elemento de la defensa de la OTAN, aunque bajo el criterio de la estrategia de respuesta flexible (como alternativa a la represalia masiva de Eisenhower) y, finalmente, confirmaba la propuesta del anterior secretario

\footnotetext{
${ }^{27}$ Ibídem, p. 118, nota al pie.

${ }^{28}$ National Security Action Memorandum Number 40: Policy Directive Regarding NATO and the Atlantic Nations (24/04/1961), John F. Kennedy Library and Museum, Boston. «http://www.jfklibrary.org/Asset-Viewer/Archives/ JFKNSF-329-015.aspx» [consultado el 4 de febrero de 2016].

${ }^{29}$ Address to Canadian Parliament, Ottawa (17/05/1961), John F. Kennedy Library and Museum, Boston. «http:// www.jfklibrary.org/Asset-Viewer/Archives/JFKPOF-034-027.aspx» [consultado el 4 de febrero de 2016].
} 
de Estado y que se estudiaría la posibilidad de establecer una fuerza naval conjunta equipada con misiles si los aliados la consideraban necesaria y útil.

Sin embargo, la MLF quedaba condicionada a que la OTAN alcanzara determinados niveles de fuerzas convencionales. La administración Kennedy reconocía que la MLF "no respondía a una verdadera necesidad militar y que su propósito era estrictamente político"30. Tampoco la Junta de Jefes de Estado Mayor era favorable, al igual que la Fuerza Aérea y el Ejército, ya que no desempeñaban ningún papel en la MLF. Dado que Kennedy no estaba dispuesto a presionar a los aliados sobre una iniciativa que había surgido en la administración anterior, la iniciativa quedó estancada.

En un NSAM del 18 de abril de $1962^{31}$, Kennedy indicaba que EEUU debería manifestar claramente su voluntad de unirse a sus aliados en la MLF y su compromiso de que la autorización para el lanzamiento se otorgaría a un órgano colectivo. El 5 de mayo los secretarios de Defensa y Estado, Robert McNamara y Dean Rusk, presentaron la propuesta en la reunión ministerial de la OTAN en Atenas, y afirmaron que EEUU ayudaría a los países aliados a adquirir misiles si estos eran destinados a la MLF.

McNamara encargó a la US Navy un estudio para detallar los problemas militares, políticos y legales que supondría la creación de una fuerza multilateral. El estudio dirigido por el vicealmirante Claude Ricketts fue terminado el 15 de junio concluyendo que una fuerza naval multilateral era viable ${ }^{32}$. Aunque se reconocía que la probabilidad de que la MLF fuera aceptada era muy baja, finalmente el 16 de junio el embajador Thomas Finletter presentó oficialmente la MLF ante el NAC.

\subsection{La crisis del Skybolt}

Como se ha anotado, el Reino Unido había abandonado el desarrollo del misil Blue Streak y se había incorporado al programa Skybolt. Sin embargo, el 24 de agosto de 1962 McNamara decidió cancelar este misil cuando los ensayos revelaron que no cumpliría los requisitos. McNamara informó el 7 de noviembre a Kennedy que el Skybolt era caro, redundante (los misiles Polaris y Minuteman cubrían aspectos similares) y, ante todo, poseía pobres prestaciones ${ }^{33}$. Cuando Kennedy preguntó sobre la reacción británica, McNamara respondió que se ocuparía de la cuestión, aunque se limitó a telefonear al ministro británico de Defensa Peter Thorneycroft para comunicarle que existía la posibilidad de que el Skybolt fuera cancelado ${ }^{34}$. McNamara llegó

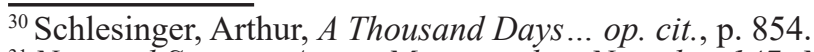

${ }^{31}$ National Security Action Memorandum Nunmber 147: NATO Nuclear Program (18/04/1962), John F. Kennedy Library and Museum, Boston. «http://www.jfklibrary.org/Asset-Viewer/OKxn4TISHEmdn4X3FQQLhw.aspx» [consultado el 4 de abril de 2016].

${ }^{32}$ Schlesinger, Arthur, A Thousand Days... op. cit., p. 854.

${ }^{33}$ Rusk, Dean y Tom Rusk, As I Saw It: a Secretary of State's memoirs, Nueva York, Norton, 1990, p. 266.

${ }^{34}$ Shapely, Deborah, Promise and Power: The Life and Times of Robert McNamara, Boston, Little, Brown and Company, 1993, pp. 242-243.
} 
a Londres el 11 de diciembre para explicar personalmente la decisión oficial; hasta entonces Thorneycroft y Macmillan solo disponían de informes sobre dificultades técnicas en el misil, por lo que ambos quedaron conmocionados ante una noticia cuyo impacto fue descrito como "si hubiera estallado un trueno sobre Londres" 35 .

Kennedy y Macmillan se encontraron en Nassau el 19 de diciembre; aunque la disuasión nuclear británica acababa de desaparecer de un plumazo, Kennedy dejó claro que no se continuaría con el Skybolt. Macmillan, por su parte, tuvo que admitir que el Reino Unido no podría asumir los costes para solucionar los fallos del misil y solicitó Polaris para equipar los futuros submarinos británicos ${ }^{36}$. Kennedy dudó a este respecto, ya que significaba la continuidad de la fuerza nuclear británica en contra de los esfuerzos por constituir la MLF, pero accedió (en caso contrario el Reino Unido modernizaría su componente nuclear fuera del control estadounidense) a cambio del compromiso de que los submarinos británicos quedaran integrados en una eventual fuerza de la OTAN y que el gobierno británico participara activamente en la creación de la MLF. A este respecto, se ha sugerido que con la desaparición repentina de la independencia británica que implicaba la cancelación del Skybolt, los EEUU encontraron el modo idóneo para forzar al Reino Unido a que integrara su componente nuclear en una fuerza multilateral; además, Kennedy confiaba en que, si se aceptaba esta circunstancia, el gobierno británico mostraría una voluntad europeísta que favoreciera su entrada en el Mercado Común Europeo ${ }^{37}$.

Kennedy y Macmillan publicaron una declaración conjunta el 20 de diciembre; entre otros aspectos, se contemplaba la creación de dos fuerzas: la primera quedaría bajo el mando de la OTAN, a la que se invitaba a participar a todos los aliados; su núcleo principal sería el Mando Aéreo Estratégico de EEUU, la V-Force y los submarinos británicos. La segunda fuerza nuclear, considerada como materialización de la MLF, sería estrictamente naval y multinacional. Kennedy presentó el acuerdo de Nassau en su discurso sobre el Estado de la Unión del 14 de enero de 1963 en los siguientes términos:

\footnotetext{
"El acuerdo de Nassau reconoce que la seguridad de Occidente es indivisible [...]. Pero también reconoce que esta es una alianza de naciones orgullosas y soberanas, y funciona mejor cuando no lo olvidamos. Reconoce además que la defensa nuclear de Occidente no es un asunto de las actuales potencias nucleares a solas, de las que Francia lo será en el futuro, y que se deben encontrar fórmulas con las que, sin aumentar los riesgos de proliferación de armas nucleares, se incremente el papel de nuestros otros socios en la planificación, dotaciones y dirección de una fuerza nuclear verdaderamente multilateral $[\ldots]^{\prime 33}$.
}

\footnotetext{
${ }^{35}$ Rusk, Dean y Tom Rusk, As I Saw It... op. cit., p. 266.

${ }^{36}$ Ibídem, p. 267.

${ }^{37}$ Vid. Cunningham, Jack, Nuclear Sharing and Nuclear Crises: A Study in Anglo-American Relations, 1957-1963, tesis doctoral, Universidad de Toronto, 2010.

${ }^{38}$ Kennedy, John Fitzgerald, Annual Message to the Congress on the State of the Union, 14 de enero de 1963. «http://www.presidency.ucsb.edu/ws/?pid=9138» [consultado el 14 de abril de 2016].
} 


\title{
1.2 La negativa de De Gaulle
}

Kennedy esperaba cohesionar la OTAN, pero no deseaba que el acuerdo de Nassau fuera interpretado como una imposición, por lo que ofreció el Polaris a Francia bajo el compromiso de su participación en la MLF. El 21 de diciembre, el secretario general de la Presidencia de la República, Etienne Burin des Roziers, recibía una propuesta para suscribir el acuerdo de Nassau ${ }^{39}$; aunque EEUU situaba a Francia al mismo nivel que el Reino Unido y, en cierto modo, creaba el Directorio Tripartito propuesto por De Gaulle y rechazado por Eisenhower, el acuerdo de Nassau fue interpretado como una muestra más de favoritismo al Reino Unido. El 14 de enero de 1963, durante una rueda de prensa, De Gaulle definió las líneas de política exterior francesa para los siguientes años. Al mismo tiempo que se oponía a la entrada del Reino Unido en el Mercado Común Europeo, rechazó la propuesta de unirse al acuerdo de Nassau y a participar en la MLF:

\begin{abstract}
"Francia ha tomado nota del acuerdo anglo-americano de las Bahamas. Tal y como es concebido, nadie se sorprenderá de que no lo suscribamos. No sería verdaderamente útil para nosotros comprar los misiles Polaris, ya que ni disponemos de submarinos para lanzarlos, ni de cabezas termonucleares con que armarlos [...]. Un día, cuando dispongamos de submarinos y de cabezas ¿cuál sería entonces la utilidad de los Polaris? En ese momento, presumiblemente, dispondremos de misiles de nuestra propia invención [...] tampoco responde al principio del que he venido hablando, que consiste en disponer de nuestra propia fuerza de disuasión. Volcar nuestros medios en una fuerza multilateral bajo mando extranjero, significaría contravenir los principios de nuestra defensa y de nuestra política. Es cierto que podríamos retener, teóricamente, la capacidad de retomar en nuestras manos, en una hipótesis suprema, nuestros elementos incorporados a la fuerza multilateral [...]. Y dado que esta fuerza multilateral conlleva necesariamente una maraña de enlaces, transmisiones e interferencias en sí misma y una envoltura de subordinaciones externas tales que, si se retirara una parte integral de ella, nos arriesgamos a paralizarla en el momento mismo en el que debería actuar. En definitiva, nos mantenemos en la decisión que hemos tomado de construir y, llegado el caso, emplear nuestra fuerza atómica" ${ }^{40}$.
\end{abstract}

Aunque los argumentos de De Gaulle se centraban en cuestiones técnicas (las cabezas nucleares francesas eran demasiado grandes para el Polaris), con su negativa expresaban su firme voluntad de mantener la autonomía frente a EEUU. El apoyo estadounidense en las guerras mundiales, aunque crucial para Francia, había llegado con retraso en ambos casos, por lo que De Gaulle estaba decidido a no repetir esta circunstancia una tercera ocasión. La propuesta de

\footnotetext{
${ }^{39}$ Bruin des Roziers, Etienne, Retour aux sources, 1962: l'année décisive, París, Plon, 1985, p. 158.

${ }^{40}$ De Gaulle, Charles, Discours et messages... op. cit., p. 258.
} 
voto unánime para autorizar el lanzamiento de los misiles implicaba que EEUU conservaría derecho a veto, por lo que para De Gaulle el verdadero propósito de aquella era absorber las fuerzas nucleares europeas y conceder a los aliados la ilusión de desempeñar un papel en la estrategia nuclear. Finalmente, el compromiso estadounidense de destinar sus submarinos a la MLF en realidad significaba "traspasar su mando de un comandante estadounidense a otro"

Francia desafiaba abiertamente a EEUU por el liderazgo en Europa y se concretaba ahora el vaticinio de Eisenhower sobre las dificultades que De Gaulle representaría para la OTAN ${ }^{42}$. El desafío francés se consolidó el 20 de enero con la firma del Tratado de Amistad y Cooperación franco-alemán, por el que ambos países se comprometían a estrechar sus relaciones en todas las áreas, incluyendo la defensa. Siguiendo la estela francesa, Canadá, Dinamarca, Noruega y Portugal rehusaron participar en la MLF. El firme rechazo francés iba a provocar, por el contario, que EEUU se decidiera a dar un impulso al proyecto. Ante una eventual oferta de asistencia nuclear francesa a Alemania, la fuerza multinacional debía aparecer como una alternativa viable $\mathrm{y}$, ante todo, económica. El objetivo era ahora contrarrestar una plausible hegemonía francesa en Europa, tanto en el aspecto político, como en el militar. Desde la perspectiva norteamericana, la creación de un bloque franco-alemán, con sus correspondientes fuerzas armadas, debilitaría la influencia de EEUU y podría conducir a una crisis en el seno de la OTAN ${ }^{43}$.

\subsection{La MLF en Europa}

Así pues, Kennedy estaba obligado a intensificar su campaña por la creación de la MLF; el 24 de enero encargó a Livingston Merchant, anterior embajador estadounidense ante la OTAN, que preparara reuniones informativas para presentar y explicar el proyecto ante los distintos jefes de gobierno europeos. E1 7 de febrero Kennedy anunciaba en una conferencia de prensa que con el acuerdo de Nassau EEUU había cumplido sus compromisos con los británicos, al tiempo que se fortalecía la OTAN con el compromiso del establecimiento de la MLF ${ }^{44}$. El día 21, Kennedy dio instrucciones a Merchant para recabar el apoyo de al menos dos de los aliados sobre la MLF. Merchant llegó en primer lugar a París, donde informó al NAC el 28 de febrero; allí, el embajador Finletter reiteró el carácter multinacional de las tripulaciones y el establecimiento de autorización unánime para el lanzamiento de los misiles y, de forma inesperada, definió la MLF como una flota de buques de superficie. Dado que el acuerdo de Nassau establecía que el Reino Unido recibiría misiles Polaris modificados para su lanzamiento desde submarinos, y a que las anteriores propuestas se basaban en estos buques, los europeos habían dado por hecho

\footnotetext{
${ }^{41}$ Ibídem, p. 258.

${ }^{42}$ Ferrell, Robert Hugh (ed.), The Eisenhower diaries... op. cit., p. 381.

${ }^{43}$ Kustnetsov, Evgeny, The Multilateral Force... op. cit.

${ }^{44}$ Grabación sonora de la conferencia de prensa en el Departamento de Estado (7 de febrero de 1963), John F. Kennedy Library and Museum, Boston. «http://www.jfklibrary.org/Asset-Viewer/Archives/JFKWHA-162.aspx» [consultado el 4 de febrero de 2016].
} 
que sería así. La nueva propuesta definía una flota formada por 25 buques de tipo mercante, equipado cada uno con ocho misiles Polaris modificados para su lanzamiento desde un nuevo tipo de silo. Se argumentó que el coste de un buque de superficie con motores diésel era menor, que podrían ser construidos en astilleros europeos y que las tripulaciones podrían adaptarse a operar con rapidez buques más sencillos que los complejos submarinos nucleares.

En París, Merchant se entrevistó con el Ministro de Asuntos Exteriores francés, Maurice Couve de Murville, quien reiteró la falta de interés de Francia en la MLF; en Roma, Merchant se reunió con el primer ministro Amintore Fanfani, quien se mostró dispuesto a participar. E1 5 de marzo, se reunió en Bruselas con el secretario general de la OTAN, el belga Paul Henri Spaak, el rey y el primer ministro de Bélgica, quienes estaban de acuerdo con las líneas generales de la MLF. Al día siguiente, Merchant llegó a Bonn, donde la MLF era vista por Adenauer como la vinculación definitiva de EEUU con la defensa de Europa y, además, abría una vía para el acceso al armamento nuclear sin implicar su posesión directa. El gobierno estadounidense era del mismo parecer pues se permitiría así a Alemania jugar un papel en el escenario nuclear, sin la necesidad de crear un componente disuasorio que "levantaría viejos fantasmas" de conversaciones terminó el 12 de marzo en Londres. Merchant constató que el principal interés de Macmillan era el establecimiento de la fuerza nuclear que recogía el acuerdo de Nassau, pues otorgaba al Reino Unido un mayor protagonismo. Merchant señaló que la MLF también estaba recogida en Nassau y estaba vinculada a la adquisición de los Polaris; además recalcó que la MLF representaba una oportunidad para desmontar el argumento de De Gaulle de que el Reino Unido carecía de mentalidad europea.

A finales de marzo de 1963 la MLF parecía quedar potencialmente formada por EEUU, Reino Unido, Italia y Alemania, los cuales habían logrado un consenso en el carácter multinacional, mando conjunto y la apertura a futuros participantes. Aunque se posponía lo referente a la autorización unánime para el lanzamiento y la sustitución de los submarinos, se acordó crear una comisión encargada de elaborar un tratado para la constitución de una Fuerza Multilateral.

En abril, Kennedy encargó un estudio destinado a valorar la capacidad de supervivencia de la flota de superficie a un ataque soviético y calibrar la posibilidad de que información sobre tecnología nuclear quedara comprometida en los buques de la MLF. El estudio de la US Navy concluyó que, en el peor de los supuestos, la URSS conseguiría eliminar siete buques; la MLF se consideraba viable, eficaz y creíble, mientras que la confidencialidad tecnológica podía ser asegurada mediante el refuerzo de los sistemas de codificación.

La todavía indeterminada postura del gobierno británico preocupaba especialmente a Kennedy, ya que sería difícil conseguir que el Congreso aprobara el Acuerdo de Nassau si el Reino Unido no cumplía con él. La indecisión británica se hizo más difícil de mantener durante

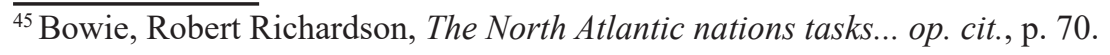


la visita a Europa que Kennedy inició el 23 de junio. En Frankfurt, Kennedy aseguró que EEUU no pretendía dominar Europa, sino que se hallaba comprometido plenamente con su defensa y que "arriesgará sus ciudades para defender las vuestras, porque necesitamos defender vuestra libertad para proteger la nuestra" ${ }^{46}$. El viaje concluyó con una reunión con Macmillan el 29 de junio. En esta entrevista Kennedy hizo patente su decepción por la escasa acogida que, salvo en la República Federal de Alemania (RFA), había recibido la MLF. Kennedy simplemente decidió no presionar a Macmillan y ambos acordaron proseguir los trabajos de forma pausada, lo que permitía al Reino Unido no emitir ninguna decisión definitiva.

Merchant y Gerard Smith iniciaron el 18 de julio en Washington una serie de reuniones con los embajadores de la RFA, Italia, Grecia y Turquía. Tras algunas semanas, Alemania e Italia propusieron establecer un grupo de trabajo con sede en París, que fue aprobado con la abstención del Reino Unido. El Grupo de Trabajo celebró su primera reunión el 11 de octubre en París. Se lograron compromisos relativos a la elección del tipo de buques, acordándose finalmente que la MLF estaría compuesta por veinticinco buques de superficie armados con ocho misiles Polaris. Los buques deberían construirse con cascos de mercantes, con los que confiaban pasar desapercibidos en rutas comerciales. Se establecería una base en el Mediterráneo y otra en el Atlántico. También se asignó un determinado porcentaje de votos a cada país para resolver cuestiones operacionales, en consonancia con la financiación aportada pero que no se aplicaría en la autorización de lanzamiento de misiles. Las contribuciones financieras serían del 35\% para EEUU y Alemania, 15\% para Italia, 10\% para Gran Bretaña y 5\% para los Países Bajos. Sobre la seguridad de las armas nucleares, se estableció que los misiles recibirían protocolos de la US Navy y que en cada buque un oficial estadounidense quedaría a cargo de los códigos de seguridad y el mantenimiento de los misiles.

El subgrupo de cuestiones militares sugirió comprobar la viabilidad de las dotaciones multinacionales en un buque ya en servicio. La idea había sido recogida en el NSAM 253 del 13 de julio de $1963^{47}$, donde se planteaba destinar una dotación multinacional a uno de los buques de la $6^{\text {a }}$ Flota, con el propósito de probar el concepto de dotación mixta. Los asesores de Kennedy informaron favorablemente y se ofreció un destructor lanzamisiles durante dieciocho meses. El subgrupo dio su aprobación el 25 de octubre, aunque el experimento se iniciaría ya durante la presidencia de Lyndon B. Johnson. En enero de 1964 se autorizó a emplear el destructor USS Biddle, rebautizado el 28 de julio como Claude V. Ricketts en honor al almirante estadounidense, ferviente defensor de la MLF, fallecido el anterior día 6. El comandante del buque, enarbolando la insignia de la OTAN, sería un estadounidense al igual que su oficial de comunicaciones, mientras que la tripulación quedaría compuesta por 8 oficiales y 164 marineros

\footnotetext{
${ }^{46}$ Schlesinger, Arthur M, A Thousand Days... op. cit., p. 884.

${ }^{47}$ National Security Action Memorandum Number 253: Multilateral Force (13 de julio de 1963); John F. Kennedy Library and Museum, Boston. «http://www.jfklibrary.org/Asset-Viewer/eeGL-UgE8kSgIM8UDmxseg.aspx» [consultado el 24 de febrero de 2016].
} 
estadounidenses y otros 14 oficiales y 166 marineros procedentes de Italia, RFA, Reino Unido, Italia, Grecia, Países Bajos y Turquía.

La dotación se completó en junio y su servicio comenzó oficialmente el 1 de julio. La tripulación no fue distribuida por nacionalidades, sino atendiendo estrictamente a sus capacidades técnicas. El buque llevó a cabo misiones rutinarias en aguas europeas, recalando en Italia, Grecia, Gran Bretaña, Países Bajos y Alemania, dando por finalizado su crucero en diciembre de 1965. El resultado se consideró satisfactorio, demostrando como un buque complejo podía ser operado de forma efectiva por hombres de diferentes naciones. Sin embargo, el experimento concluyó al mismo tiempo que la MLF caía en la indiferencia de la mayoría de los implicados.

\section{La disolución de la MLF}

Aunque Lyndon B. Johnson no compartía personalmente los presupuestos de la MLF, decidió dar continuidad a los proyectos de Kennedy y aprobó a principios de diciembre las recomendaciones que se le hicieron sobre la $\mathrm{MLF}^{48}$. En febrero de 1964, Peter Thorneycroft llegó a EEUU para entrevistarse con Johnson y explicar que el Reino Unido seguía indeciso sobre su participación en la MLF y que se estaban considerando otras variantes para una fuerza multilateral. Johnson aceptó que la decisión británica no fuera definitiva hasta después de las elecciones de octubre. Las propuestas británicas fueron presentadas formalmente al Grupo de Trabajo el 2 de julio, momento en el que el resto de países habían aceptado la mayor parte de las propuestas norteamericanas y en el que, para desesperación británica, la RFA se había convertido en el principal valedor de la MLF.

El 12 de junio, el canciller alemán Ludwig Erhard se había entrevistado con Johnson en Washington; la sintonía que demostraron pareció que se desarrollara como si "existiera un tratado germano-estadounidense tácito, paralelo al explícito tratado germano-francés" $" 49$. Johnson informó a Erhard que se estaban esperando los resultados de las elecciones británicas mientras se daba tiempo a los italianos para apuntalar su gobierno de coalición, antes de que ambos dieran su apoyo a la MLF a principios de 1965.

Sin embargo, en las elecciones británicas se produjo la victoria del partido laborista y Harold Wilson fue nombrado primer ministro el día 16 de octubre. Los laboristas se habían opuesto tradicionalmente a la fuerza nuclear británica, defendían amplios recortes en defensa y el mismo Wilson había criticado directamente la MLF. Ese mismo mes el gobierno italiano

\footnotetext{
${ }_{48}$ Priest, Andrew, "The President, the «Theologians» and the Europeans: The Johnson Administration and NATO Nuclear Sharing", en The International History Review, vol. 33, 2 (2011), pp. 257-277. DOI: http://dx.doi.org/10 .1080/07075332.2011.555437.

49 “Boletín del día: Erhard a Washington", en La Vanguardia española, 11 de junio de 1964, p. 15.
} 
confirmó que su continuidad era muy precaria y que no estaría en condiciones de firmar un acuerdo antes de marzo de 1965.

Así pues, a finales de 1964 la MLF parecía reducida a una empresa germanoestadounidense; unas ambivalentes declaraciones de Erhard sobre un acuerdo bilateral tuvieron que ser inmediatamente corregidas. Quedaba claro que la posibilidad de que se "permitiera a un dedo alemán situarse sobre un botón nuclear" ${ }^{\prime 50}$ despertaba aprensiones de todo tipo; en Francia, George Pompidou declaraba que se revisaría el tratado franco-alemán si la MLF derivaba en una alianza militar germano-americana, mientras que el 22 de noviembre, durante una visita a Estrasburgo, De Gaulle emplazó al canciller Erhard a construir "una Europa europea y organizar una Europa capaz de defenderse por sí misma" ${ }^{51}$. Durante la visita de Dean Rusk a París el 14 de diciembre, De Gaulle le aseguró que no aceptaría que Alemania dispusiera, directa o indirectamente, de armamento nuclear y, ese mismo mes, Couve de Murville declaraba en el consejo ministerial de la OTAN que la MLF perjudicaría la reunificación alemana. Resultaba evidente que el apoyo de un país a la MLF supondría enturbiar sus relaciones con Francia.

\subsection{Epílogo: la ANF}

Aunque la fuerza nuclear británica era considerada marginal y redundante en la estrategia estadounidense, el Reino Unido era el miembro europeo más importante con el que podía contar la MLF, pero también era el más indeciso. Si no se había rechazado abiertamente la participación en un proyecto que significaba perder el control de las armas nucleares propias, era porque el Reino Unido no quería perder su categoría de gran potencia, disminuida tras la desaparición del imperio británico, ni tampoco dar motivo para romper los estrechos lazos bilaterales con EEUU ${ }^{52}$. Una vez en el poder, Wilson comprendió que las armas nucleares otorgaban ese perdido peso internacional, por lo que decidió lograr un compromiso que le permitiera recortar los gastos de defensa preservando la disuasión nuclear británica. Por otro lado, debido a sus declaraciones durante la campaña electoral, le resultaba imposible apoyar la creación de la MLF, por lo que en noviembre propuso como alternativa, la creación de una estructura mayor que englobaría a la MLF, la Fuerza Nuclear Atlántica (Atlantic Nuclear Force, ANF), bajo mando de la OTAN en lugar de quedar a disposición del SACEUR. Estaría formada por los bombarderos de la $V$-Force y del SAC, los submarinos de EEUU y Reino Unido y la flota de la MLF, abierta a la participación de los países que lo deseasen sin convertirse en requisito para formar parte de la ANF.

\footnotetext{
$\overline{{ }^{50} \text { Henri, Pierre, “ }}$ Les Travaillistes préfèrent les Sept aux Six”, en Le Monde Diplomatique, noviembre de 1964, p. 4.

${ }_{51}^{5}$ De Gaulle, Charles, Discours et messages... op. cit., p. 258.

${ }^{52}$ Widén, J. J. y Jonathan Colman, "Lyndon B. Johnson, alec Douglas-home, Europe and the Nato multilateral force, 1963-64", en Journal of Transatlantic Studies, vol. 5, 2 (2008), p. 193. DOI: http://dx.doi. org/10.1080/14794019908656863.
} 
Los bombarderos y submarinos que se incluyeran en la ANF pasarían a ser de propiedad y titularidad conjunta, pero conservando sus tripulaciones nacionales. De este modo, el Reino Unido podría contribuir mediante aviones y buques de los que disponía, o dispondría en un futuro, sin contribuir a la creación ex profeso de la MLF, cambios que alteraban sustancialmente el espíritu de esta. De hecho, la historiografía posterior ha señalado que esta propuesta tuvo la clara intención de frustrar en todo lo posible las iniciativas sobre la MLF, en clara represalia por la puesta en entredicho del componente nuclear británico; para el gobierno británico, conservador o laborista, la posesión de un componente disuasorio propio era un factor decisivo en el mantenimiento del status de gran potencia, por lo que la ANF era útil para aparentar una independencia nuclear que no existía ${ }^{53}$.

Para preparar la próxima visita de Wilson, Johnson convocó a McNamara, Rusk, Bundy y Ball, quienes le informaron que la MLF era el principal escollo tanto para concluir un tratado de no proliferación nuclear con la URSS, como una de las razones del alejamiento de Francia. Buena parte del Congreso se mostraba contrario, y dado que el apoyo europeo no era tan amplio como se le había hecho creer, Johnson decidió no promover la MLF en el Congreso.

Wilson llegó a la Casa Blanca el 7 de diciembre y planteó la cuestión de la MLF tras detallar los inconvenientes que el Reino Unido encontraba en la cuestión y entregó la propuesta de creación de la ANF. Johnson convocó a sus asesores para estudiar el modo de proceder sobre la MLF-ANF y pidió una fórmula honrosa que permitiera a EEUU retirarse del proyecto. Cuando la delegación británica llegó, Johnson dejó que Wilson defendiera su plan sobre la ANF y, en una jugada magistral, anunció que EEUU apoyaría cualquier plan que contara con el apoyo de una Europa unida, desprendiéndose así de toda responsabilidad sobre la formación de una fuerza multilateral. Los aliados europeos acogieron con satisfacción la supresión de todo apremio estadounidense, pero ninguno de ellos se puso al frente de un nuevo plan, lo que dejaba a la ANF como única fórmula multilateral. Los mismos países que se habían quejado de ser presionados, acusaron a EEUU por su pérdida de interés. Johnson refirmó sus compromisos durante una conferencia de prensa del 16 de enero de $1965^{54}$, pero también que mantendría su postura. Con habilidad y cortesía, Johnson se había desecho del problema heredado que suponía la $\mathrm{MLF}^{55}$.

En la RFA, Erhard, Schroeder y Von Hazle, se sintieron defraudados con el aparente abandono estadounidense, pues habían sido firmes defensores de la MLF ante el parlamento alemán. La flota de superficie era la única forma de contribución alemana a una fuerza multilateral, y la ANF era un sucedáneo, pues su núcleo estaría compuesto por submarinos y

\footnotetext{
${ }_{53}$ Vid. Middeke, Michael, "Anglo-American Nuclear Weapons Cooperation After the Nassau Conference: The British Policy of Interdependence", en Journal of Cold War Studies, vol. 2, 2 (2000), pp. 69-96. DOI: https://doi. org/10.1162/15203970051032318.

${ }^{54}$ Johnson, Lyndon B., The President's News Conference at the LBJ Ranch (16 de enero de 1965). «http://www. presidency.ucsb.edu/ws/?pid=26930» [consultado el 17 de marzo de 2016].

${ }_{55}^{5}$ Priest, Andrew, "The President, the «Theologians..." op. cit.
} 
bombarderos, de los que la RFA carecía. Gran Bretaña presentó formalmente la ANF ante el NAC en enero de 1965 y el 6 de marzo Wilson visitó la RFA; aunque Erhard reconoció que la MLF había perdido ya todo impulso, sostuvo que esta coincidía con las aspiraciones alemanas. Wilson y Erhard tan solo acordaron iniciar conversaciones con otros países para combinar los planes de la MLF y la ANF.

Según las expectativas se difuminaban, los aliados perdieron interés en la idea. La URSS había dejado claro que el precio por lograr un tratado de no proliferación nuclear era la fuerza multilateral, fuera la ANF o la MLF. Johnson, ocupado por la guerra en Vietnam, no mencionó el tema durante la visita de Erhard en junio. Cuando ese mismo mes se pidió a la Embajada estadounidense que durante la recalada del USS Ricketts en Inglaterra el comandante del buque no hiciera mención a una fuerza multilateral en sus declaraciones a la prensa, el desencanto británico fue manifiesto.

Tras su victoria en las elecciones de octubre, Erhard confirmó su apoyo a la MLF; sin embargo, Alemania era entonces el único país que consideraba seriamente la fuerza multilateral. Erhard visitó EEUU en diciembre y regresó convencido de que Vietnam era la principal preocupación estadounidense y que la MLF se aparcaría por tiempo indefinido. Con la adhesión en 1968 de Alemania al tratado de No Proliferación Nuclear, se cerraron las aspiraciones nucleares alemanas y toda posibilidad de que la MLF pudiera materializarse ${ }^{56}$.

\section{Conclusiones}

La MLF colapsó desde su interior; el fracaso se debió simplemente a que el proyecto estaba viciado desde su concepción, ya que ni siquiera su propósito declarado, mejorar la capacidad defensiva de la OTAN mediante una fuerza compartida, era cierto "cuando el 95\% de las cabezas nucleares disponibles seguía bajo control estadounidense" ${ }^{57}$. La MLF fue ideada en un intento de cohesionar a la OTAN reemplazando el deseo de crear o desarrollar componentes nucleares nacionales. Se estimaba que, si se lograba que los aliados participaban en fuerza nuclear unificada, con las correspondientes responsabilidades sobre la planificación, operación y estrategia, la necesidad de disponer de armas nucleares propias quedaría satisfecha ${ }^{58}$.

Sin embargo, para algunos autores, el proyecto de una fuerza multilateral se encontraba viciado de origen, ya que su verdadero propósito era el deseo de mantener la hegemonía que

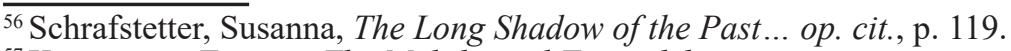

${ }^{57}$ Kustnetsov, Evgeny, The Multilateral Force debates... op. cit.

${ }^{58}$ Withers, Christopher, The multilateral force... op. cit., p. 96.
} 
la superioridad nuclear y tecnológica otorgaba a EEUU sobre sus aliados ${ }^{59}$. Bajo la apariencia formal del deseo de cohesionar a la OTAN, subyacía la negativa de EEUU a reconocer que se había perdido el monopolio de la defensa occidental; incapaces de revertir esta situación, tres administraciones estadounidenses trataron de retener el control de la totalidad de las armas nucleares occidentales, aspecto este que nadie más estaba dispuesto a aceptar. En última instancia, la posterior exasperación de EEUU se debió a su incapacidad para asumir que de haberse producido, esta situación hubiera supuesto prolongar un estado de subordinación entre supuestos iguales ${ }^{60}$.

Por otro lado, la MLF debía contribuir a un compromiso mutuo en el mantenimiento de una disuasión global creíble; si la represalia masiva promovida por Eisenhower no resultaba del todo creíble en el caso de un conflicto limitado a Europa, con la MLF los aliados lograrían participar, aunque fuera parcialmente, en la estrategia nuclear global y obtener así seguridades sobre e1 compromiso estadounidense. En compensación, la OTAN debía asumir el incremento de fuerzas convencionales que implicaba la respuesta flexible de la administración Kennedy.

Sin embargo, si con esta nueva estrategia EEUU lograba un mayor número de opciones, las de los europeos disminuían en la misma proporción. Cualquier enfrentamiento en suelo europeo, aunque comenzara al más bajo nivel, significaba una escalada que conducía al uso de armas atómicas; en este escenario, la participación de la MLF se hacía necesariamente en un conflicto sobre suelo europeo, en el que ambas superpotencias tenían la opción de mantener sus territorios como "santuarios privilegiados" $"$. En estas circunstancias, era imposible lograr un consenso en una estrategia conjunta.

Si el proyecto de fuerza multilateral no fue rechazado desde el inicio fue debido a que los EEUU trataron de resolver varios problemas mediante una única solución. En primer lugar, se trataba de obtener el control de los componentes disuasorios de Francia y el Reino Unido para mantener una estrategia; íntimamente relacionado con este punto se encontraba el recelo que despertaba De Gaulle y la empecinada autonomía militar que estaba empeñado en mantener, aun si para ello debía enfrentarse con EEUU. Algunos estudios historiográficos señalan que la MLF, y más concretamente las presiones estadounidenses, presentadas como injerencias inaceptables, fueron aprovechadas por los más recalcitrantes gaullistas para favorecer sus posturas anti-atlantistas y, especialmente, anti-británicas ${ }^{62}$.

El caso francés representaba un peligroso ejemplo a seguir por otros países europeos, supuesto especialmente aterrador si se materializaba en un componente nuclear propio para Alemania, tan solo 15 años después del fin de la Segunda Guerra Mundial. En su último estado

\footnotetext{
${ }^{59}$ Baum, Keith, "Treating the Allies Properly: The Eisenhower Administration, NATO, and the Multilateral Force", en Presidential Studies Quarterly, vol. 13, 1 (1983), p. 85.

${ }^{60}$ Ibidem, p. 85.

${ }^{61}$ Withers, Christopher, The multilateral force... op. cit., p. 98.

${ }^{62}$ Vid. Barbier, Colette, "La force multilatérale dans le débat atomique français", en Revue d'histoire diplomatique, vol. 107, 1 (1993), pp. 55-89.
} 
evolutivo, la MLF estaba más cerca de ser una fórmula para satisfacer las aspiraciones alemanas de reconocimiento, que de la idea original de integrar las armas nucleares francesas y británicas. Abundando en las contradicciones de la MLF, la reciente historiografía ha señalado que fueron los aliados de Alemania quienes impusieron su rearme como condición para que el país fuera de nuevo aceptado en la comunidad internacional; sin embargo, no parecían estar muy dispuestos a aceptar que el rearme alemán incluyera armas nucleares, en plena era nuclear ${ }^{63}$.

Finalmente, el único aspecto militar que podía cubrir la MLF era el de poner armas nucleares a disposición inmediata de la OTAN. En realidad, ni siquiera este punto podía ser solucionado convenientemente ya que los MRBM, incluso si se empleaban de forma limitada, implicaban un conflicto nuclear sobre suelo europeo que, de forma previsible, acabaría degenerando en una guerra nuclear global.

Debatida dentro y fuera de la OTAN, los esfuerzos estadounidenses por llevarla adelante fueron intermitentes; a pesar de su posición de líder, EEUU nunca logró un acuerdo al olvidar "que la OTAN no consistía en una unidad política, sino en una alianza militar"64 cuyo mando centralizado entraba en conflicto con la soberanía nacional, a cuya renuncia no estaba dispuesto ninguno de los aliados. El único legado de la MLF fue la prolongación del debate sobre el control de las armas nucleares, incluso cuando ya se había renunciado al proyecto; en lugar de aportar una mayor cohesión, la Fuerza Multilateral produjo "el efecto inverso": un elevado grado de discordancia en el seno de la OTAN ${ }^{65}$.

\footnotetext{
${ }^{63}$ Schrafstetter, Susanna, The Long Shadow of the Past... op. cit., p. 119.

${ }^{64}$ Kissinger, Henry, The troubled partnership: a re-appraisal of the Atlantic Alliance, Nueva York, McGraw-Hill, 1964, p. 152.

${ }^{65}$ Withers, Christopher, The multilateral force... op. cit., p. 101.
} 